\title{
Reusable Oxidation Catalysis Using Metal-Monocatecholato Species in a Robust Metal-Organic Framework
}

\author{
Honghan Fei, ${ }^{\dagger}$ JaeWook Shin, ${ }^{\ddagger}$ Ying Shirley Meng, ${ }^{\ddagger}$ Mario Adelhardt, ${ }^{\S}$ Jörg Sutter, ${ }^{\S}$ Karsten Meyer, ${ }^{\S}$ \\ and Seth M. Cohen ${ }^{* \dagger}$ \\ ${ }^{\dagger}$ Department of Chemistry and Biochemistry and ${ }^{\star}$ Department of NanoEngineering, University of California, San Diego, La Jolla, \\ California 92093, United States \\ ${ }^{\S}$ Department of Chemistry and Pharmacy, Inorganic Chemistry, Friedrich-Alexander University Erlangen - Nürnberg (FAU), \\ Egerlandstrasse 1, 91058 Erlangen, Germany
}

Supporting Information

ABSTRACT: An isolated metal-monocatecholato moiety has been achieved in a highly robust metal-organic framework (MOF) by two fundamentally different postsynthetic strategies: postsynthetic deprotection (PSD) and postsynthetic exchange (PSE). Compared with PSD, PSE proved to be a more facile and efficient functionalization approach to access MOFs that could not be directly synthesized under solvothermal conditions. Metalation of the catechol functionality residing in the MOFs resulted in unprecedented $\mathrm{Fe}$ monocatecholato and Cr-monocatecholato species, which were characterized by X-ray absorption spectroscopy, X-band electron paramagnetic resonance spectroscopy, and ${ }^{57} \mathrm{Fe}$

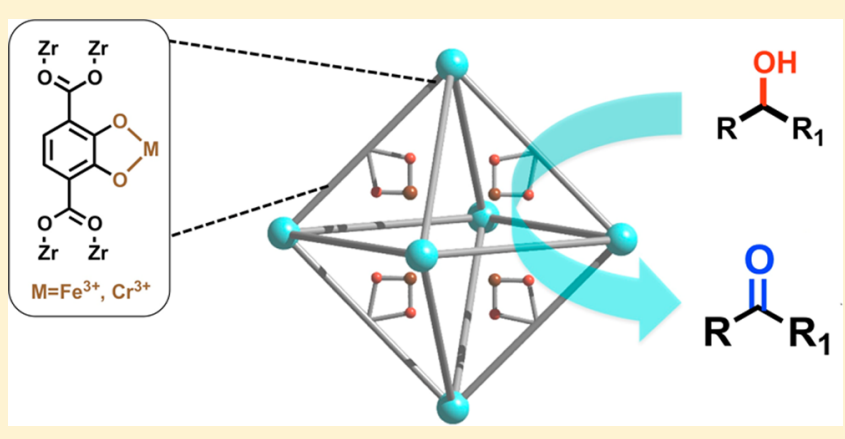
Mössbauer spectroscopy. The resulting materials are among the first examples of $\mathrm{Zr}(\mathrm{IV})$-based UiO MOFs (UiO = University of Oslo) with coordinatively unsaturated active metal centers. Importantly, the Cr-metalated MOFs are active and efficient catalysts for the oxidation of alcohols to ketones using a wide range of substrates. Catalysis could be achieved with very low metal loadings (0.5-1 mol \%). Unlike zeolite-supported, Cr-exchange oxidation catalysts, the MOF-based catalysts reported here are completely recyclable and reusable, which may make them attractive catalysts for 'green' chemistry processes.

\section{INTRODUCTION}

Metal-organic frameworks (MOFs) are a class of microporous crystalline materials gathering substantial attention not only for their vast array of structures ${ }^{1}$ but also due to their applications in gas storage, ${ }^{2}$ separations, ${ }^{3}$ catalysis, ${ }^{4}$ molecular sensing, ${ }^{5}$ and drug delivery. ${ }^{6}$ Accessible and coordinatively unsaturated metal sites residing in MOFs are desirable for gas or substrate binding within the pores, opening up a range of technological applications from improved gas storage to catalysis. ${ }^{7-9}$ In most cases, it is challenging to obtain free metal-binding sites via direct solvothermal synthesis, though some sites (e.g., carboxylic groups) have been obtained for a small number of MOFs. ${ }^{10,11}$ Postsynthetic methods are synthetic alternatives to obtain metal-binding sites in MOFs. These include methods such as postsynthetic modification (PSM), ${ }^{12-15}$ postsynthetic deprotection (PSD), ${ }^{16,17}$ and postsynthetic exchange (PSE) (also termed SALE $=$ solvent-assisted linker exchange $){ }^{18-25}$ Despite the increased use of these synthetic methods, to the best of our knowledge, no report has described the use of PSE to introduce metal-chelating groups into MOFs.

Catechol is one of the most widely used chelators in coordination chemistry. ${ }^{26}$ The standard solution coordination chemistry of catechol ligands generally leads to coordinatively saturated bis(catecholato) or tris(catecholato) homoleptic species. $^{27,28}$ Immobilized catechol ligands in MOFs provide a platform for isolating highly unsaturated mono(catecholato) metal complexes. Recently, polymeric materials bearing metalcatecholate groups have been studied in porous organic polymers using a cobalt-catalyzed acetylene trimerization strategy. $^{29-31}$ However, studies of such catalytically active metalated-catechol functionalities in MOFs are very rare. Nguyen and co-workers used PSM to incorporate single-site vanadyl(monocatecholate) moieties into two MOFs; however, the efficiency of these catalysts was limited, largely due to low metal loadings and framework instability. 32,33

Previously, photochemically driven PSD of 2-nitrobenzyl protection groups was used to generate free catechol groups in a UMCM (UMCM = University of Michigan Crystalline Material) framework. ${ }^{16}$ Herein, we employed two fundamentally different approaches (i.e., PSD and PSE) to synthesize the first highly robust $\mathrm{MOF}$ ( $\mathrm{UiO}-66, \mathrm{UiO}=$ University of Oslo) bearing isolated monocatecholato metal sites on the strut of organic linkers. Both strategies result in good loading of metal species, ${ }^{32}$ while maintaining the high porosity of the MOFs and accessibility to the open metal sites. In particular, PSE allows

Received: November 14, 2013

Published: March 5, 2014 
Scheme 1. Synthesis of UiO-66-CAT via PSD and PSE

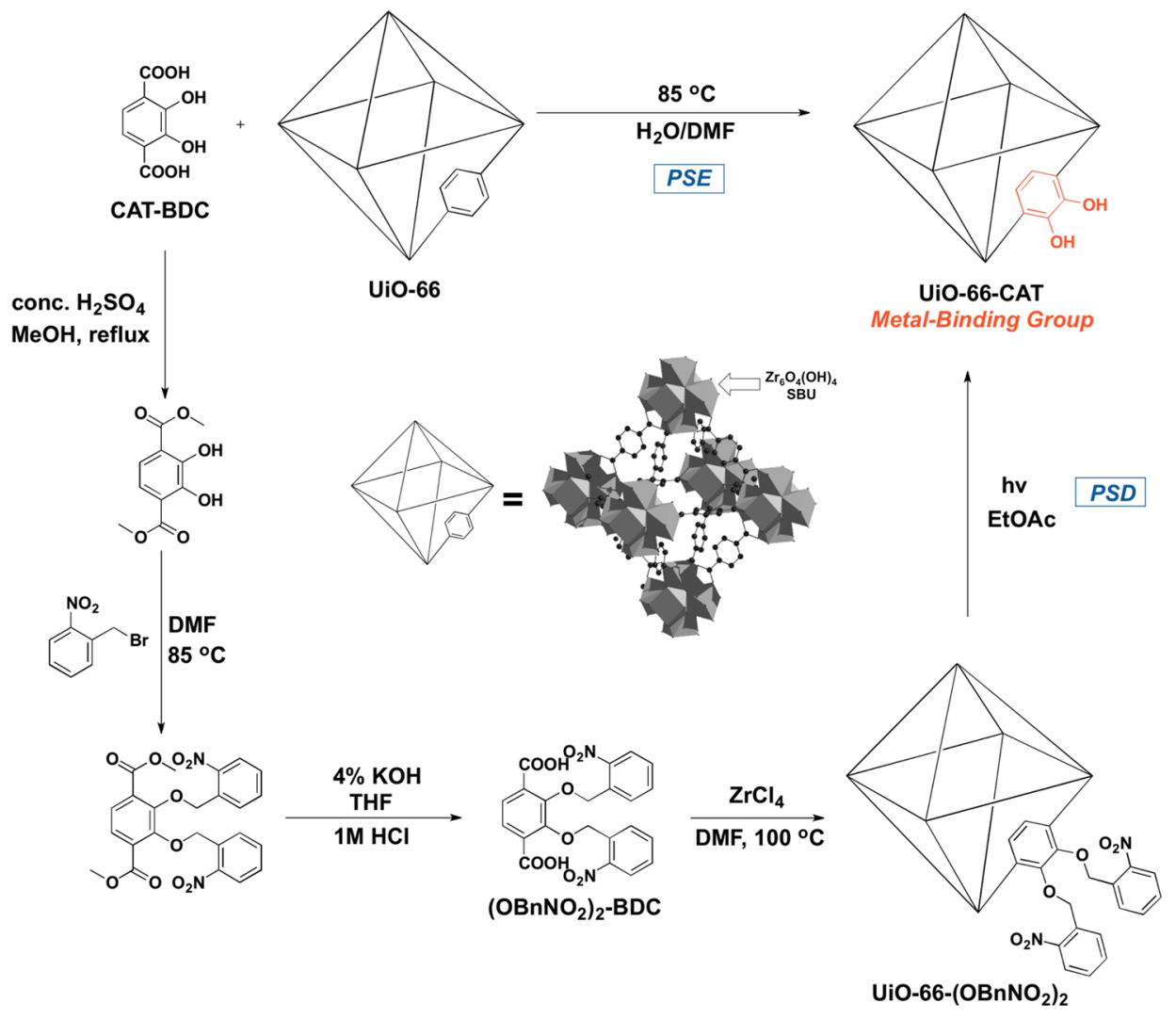

good control over the number of metal-binding sites and for a higher density of catalytic sites when compared to PSD. The metal-catecholate coordination environment ( $\mathrm{Fe}, \mathrm{Cr}$ ) was characterized by X-ray absorption spectroscopy (XAS) and diffuse reflectance UV-vis electronic spectroscopy. To the best of our knowledge, this is the first example of these $\mathrm{Zr}(\mathrm{IV})$ based MOFs decorated with a coordinatively unsaturated and catalytically active metal site. The functionalized UiO-66 platform containing metal-catecholate species was further employed as an oxidation catalyst. The immobilized $\mathrm{Cr}$ (III)monocatecholato $\mathrm{UiO}$ materials exhibit efficient catalysis of secondary alcohol oxidation with several substrates using $t$-butyl hydroperoxide (TBHP) or hydrogen peroxide $\left(\mathrm{H}_{2} \mathrm{O}_{2}\right)$ as a sacraficial oxidant. This is a rare example using MOFs to produce a nonprecious metal catalyst that is highly recyclable, thereby holding promise for "green" chemistry applications.

\section{RESULTS AND DISCUSSION}

Synthesis of UiO-66-CAT via PSD. 2,3-Bis((2nitrobenzyl)oxy)-1,4-benzene dicarboxylic acid ((OBnNO) $)_{2}$ bdc) was synthesized as previously described (Scheme 1). ${ }^{16}$ UiO-66- $\left(\mathrm{OBnNO}_{2}\right)_{2}$ was prepared using solvothermal conditions containing a 1:1 molar ratio mixture of $\left(\mathrm{OBnNO}_{2}\right)_{2}$-bdc and unfunctionalized 1,4-benzene dicarboxylic acid (bdc) with $\mathrm{ZrCl}_{4}$ and acetic acid (as a modulator) at $100{ }^{\circ} \mathrm{C}$ in DMF for $24 \mathrm{~h}$. The resulting off-white crystalline solids were washed with copious amounts of DMF and methanol, followed by activation under vacuum. Examination by ${ }^{1} \mathrm{H}$ NMR spectroscopy upon digestion of MOFs with $\mathrm{HF}$ in $d^{6}$-DMSO revealed bdc, $\left(\mathrm{OBnNO}_{2}\right)_{2}$-bdc, and a mix of mono- and fully deprotected $\left(\mathrm{OBnNO}_{2}\right)_{2}$-bdc (Figure 1). Within the resulting solids, $\sim 70 \%$ of $\left(\mathrm{OBnNO}_{2}\right)_{2}$-bdc had a single nitrobenzyl group cleaved, and

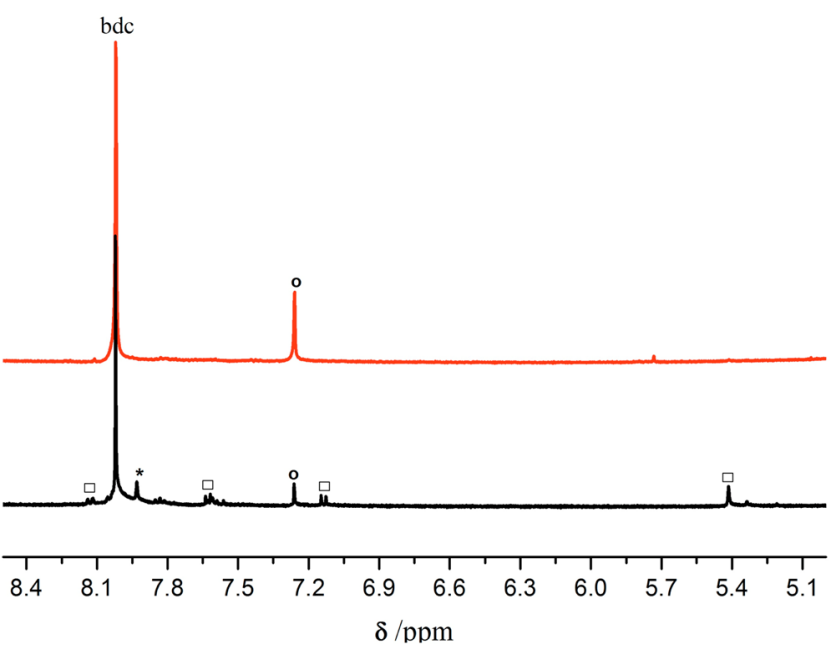

Figure 1. ${ }^{1} \mathrm{H}$ NMR of UiO-66-( $\left(\mathrm{OBnNO}_{2}\right)_{2}$ (top) and UiO-66-CAT (via PSD) (bottom). $\left(\mathrm{OBnNO}_{2}\right)(\mathrm{OH})$-bdc with a single nitrobenzyl group cleaved is labeled with squares, catbdc is labeled with circles, and DMF solvent is labeled with asterisks. The bdc ligand is also labeled in the spectra.

$25 \%$ were completely cleaved to catbdc (Scheme 1). Powder Xray diffraction (PXRD) confirmed the resulting MOFs adopt the UiO-66 framework (Figure 2). Irradiated with $365 \mathrm{~nm}$ light for $24 \mathrm{~h}, \mathrm{UiO}-66-\left(\mathrm{OBnNO}_{2}\right)_{2}$ was found to achieve nearly quantitative deprotection to yield UiO-66-CAT (via PSD). ${ }^{16,34}$ ${ }^{1} \mathrm{H}$ NMR of digested MOFs show a molar ratio of 2.2:1 (bdc:catbdc), indicating that $\left(\mathrm{OBnNO}_{2}\right)_{2}$-bdc is less effectively incorporated into $\mathrm{UiO}-66$ than bdc (Figure 1). Electrospray ionization-mass spectrometry (ESI-MS) of digested UiO-66- 

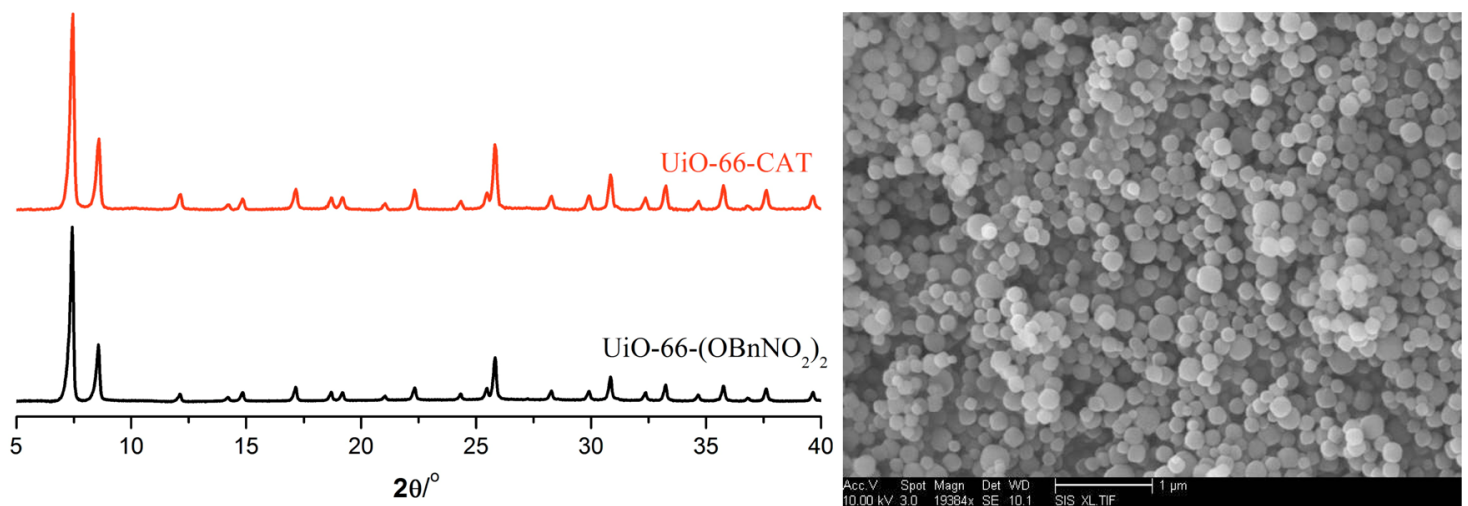

Figure 2. PXRD of UiO-66-(OBnNO$\left.)_{2}\right)_{2}$ and UiO-66-CAT (via PSD) (left). SEM image of UiO-66-CAT (via PSD) (right).

CAT (via PSD) further confirmed the presence of both bdc and catbdc in PSD product (Figure S2). PXRD suggested UiO-66CAT (via PSD) maintained the same underlying framework structure as $\mathrm{UiO}-66-\left(\mathrm{OBnNO}_{2}\right)_{2}$ (Figure 2). Scanning electron microscopy (SEM) suggested high phase purity with monodisperse, spherical particles $(\sim 150-200 \mathrm{~nm}$, Figure 2$)$.

Synthesis of UiO-66-CAT via PSE. Recently, PSE has become a facile and efficient strategy to functionalize MOFs under mild conditions. ${ }^{18-22,35}$ More importantly, using PSE to introduce a metal-chelating group into a robust MOF has not been investigated. Herein, we expanded PSE as a general functionalization method to introduce catechol groups into the UiO-66 lattice.

UiO-66 was synthesized in DMF containing a 1:1 molar ratio mixture of bdc and $\mathrm{ZrCl}_{4}$ with acetic acid (as a modulator) at $120{ }^{\circ} \mathrm{C}$ for $24 \mathrm{~h}$. The resulting crystalline solids were washed with copious amounts of $\mathrm{MeOH}$ and activated under vacuum. PSE was performed by exposing UiO-66 powder to a DMF/ $\mathrm{H}_{2} \mathrm{O}$ solution of catbdc for $2 \mathrm{~d}$ at $85{ }^{\circ} \mathrm{C}$. After separating the solids by centrifugation and washing with fresh $\mathrm{MeOH}$, the presence of catbdc in $\mathrm{UiO}-66$ was confirmed by ${ }^{1} \mathrm{H}$ NMR of the MOFs digested with dilute $\mathrm{HF}$ in $\mathrm{CD}_{3} \mathrm{OD}$ (Figure 3). The degree of functionalization was controllable based on the amount of catbdc present in the starting solution (from $18 \%$ to $75 \%$, using 0.5 to $\sim 5$ equiv of catbdc, Figure S3). ESI-MS of digested UiO-66-CAT (via PSE) further confirmed catbdc in the PSE product (Figure S4). Further increasing the ratio of catbdc in the precursor solution did not significantly enhance the incorporation; however, incubating the $75 \%$ functionalized UiO-66-CAT (via PSE) with a fresh catbdc solution for a second round of PSE gave $>90 \%$ incorporation of catbdc. Importantly, the high crystallinity of exchanged UiO-66-CAT (via PSE) was confirmed by PXRD (Figure 3). As shown by SEM, UiO-66-CAT containing 75\% catbdc (via PSE) exhibited a homogeneous $\mathrm{UiO}$ particle morphology, with no evidence of any other crystalline or amorphous phase (Figure S5). Attempts to directly synthesize UiO-66-CAT under numerous solvothermal conditions were unsuccessful, including attempts using a mixed ligand strategy with bdc and catbdc in an equimolar ratio (Table S3).

To confirm that catbdc was incorporated into the $\mathrm{UiO}$ framework (and not just included in the pores), experiments were performed examining the supernatant of the reaction mixture. PSE between UiO-66 and catbdc was performed in $\mathrm{D}_{2} \mathrm{O}$, and ${ }^{1} \mathrm{H}$ NMR of the supernatant indicated the presence of released bdc after $24 \mathrm{~h}$ of PSE at $85^{\circ} \mathrm{C}$ (Figure S6). UiO-66 in $\mathrm{D}_{2} \mathrm{O}$ in the absence of catbdc did not show any leaching of
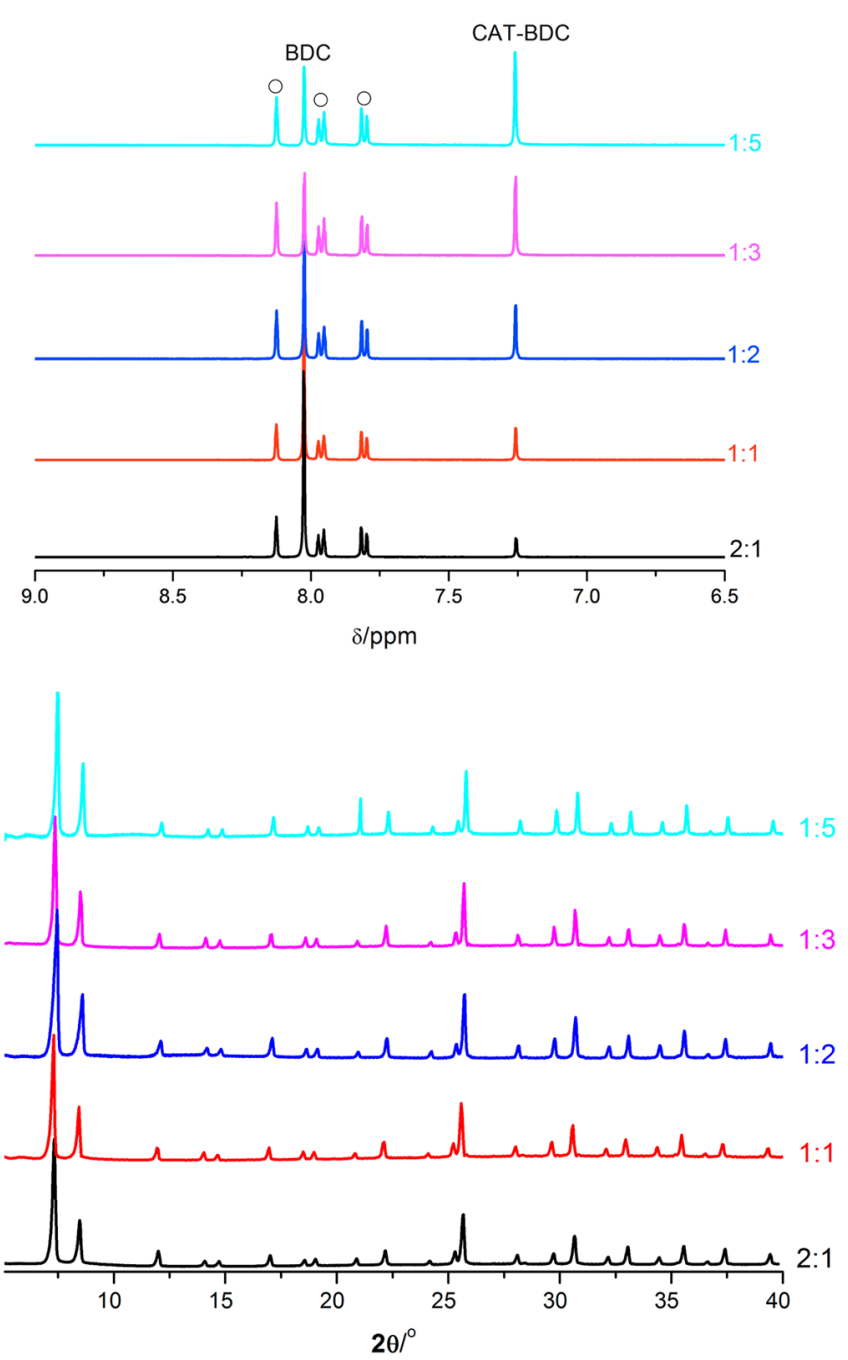

Figure 3. ${ }^{1} \mathrm{H}$ NMR (top) and PXRD (bottom) of digested UiO-66CAT synthesized by PSE with 1 equiv of UiO-66 exposed to 0.5 (black), 1 (red), 2 (blue), 3 (magenta), or 5 (cyan) equiv of catbdc. During digestion of the MOFs for ${ }^{1} \mathrm{H}$ NMR analysis, 1 equiv of Br-bdc (denoted with circles) was added as a quantitative internal standard.

bdc under the same experimental conditions. In addition, UiO66-CAT (via PSE) exhibited Brunauer-Emmett-Teller (BET) surface areas in the range of $968 \pm 27 \mathrm{~m}^{2} / \mathrm{g}$ to $1206 \pm 11 \mathrm{~m}^{2} / \mathrm{g}$ (depending on the degree of catbdc incorporation), which is close to the BET surface area of pristine UiO-66 (1403 \pm 28 $\mathrm{m}^{2} / \mathrm{g}, \mathrm{N}_{2}$ gas at $77 \mathrm{~K}$ ) (Table $\mathrm{S} 1$ ), again excluding the possibility 
that the catbdc is included in and blocking the pores of the MOF. During digestion analysis of these MOFs, 1 equiv (with respect to the total bdc content of the MOFs prior to PSE) of internal standard (2-bromoterephthalic acid, Br-bdc) was added. The NMR analysis (Figure 3 ) shows that the combined amount of all bdc linkers in the MOF is unchanged throughout PSE process. Based on these NMR and BET observations, along with high degree of catbdc incorporation, the data support a ligand metathesis process and argue against simple inclusion of catbdc into the MOF pores or attaching onto the particle surfaces. Finally, it is also important to note that the PSE process could also be carried out at room temperature, with an expectedly lower degree of catbdc functionalization, which suggests a solid-state exchange mechanism and argues against a dissolving/reforming process.

Both PSD and PSE appear to be viable strategies to incorporate catechol species into the robust UiO-66 framework. Compared with PSD, PSE exhibited two advantages for functionalization. First, as demonstrated above, PSE could be readily controlled to modulate the degree of catechol functionalization. However, PSD only allowed for $<30 \%$ functionalization of catbdc into the UiO-66 lattice, perhaps due to the steric influence of the $\left(\mathrm{OBnNO}_{2}\right)_{2}$-bdc precursor. Increasing the $\left(\mathrm{OBnNO}_{2}\right)_{2}$-bdc:bdc ligand ratio in the solvothermal synthesis led to incomplete PSD or even appearance of an amorphous phase from the reaction mixture. Second, the PSD strategy required a three-step synthesis of $\left(\mathrm{OBnNO}_{2}\right)_{2}$-bdc, a MOF synthesis step, and a photochemical PSD step as well with an overall yield of $\sim 35 \%$ (Scheme 1). In contrast, UiO-66-CAT using PSE was prepared in only two steps (i.e., UiO-66 synthesis and the PSE reaction) giving $>90 \%$ yield. Overall, the PSE strategy to incorporate the catechol units into UiO-66 proved to be a more convenient, efficient, and controllable approach.

Metalation of UiO-66-CAT with Fe. UiO-66-CAT prepared via the PSD approach described above (Scheme 1) was metalated in a postsynthetic manner with Fe. The metalation of the catechol units residing in the framework was performed by incubating UiO-66-CAT (via PSD) in an aqueous solution of $\mathrm{Fe}\left(\mathrm{ClO}_{4}\right)_{3}$, whereupon the particles became dark-brown within several minutes. After $24 \mathrm{~h}$, UiO66-FeCAT was washed extensively with fresh $\mathrm{MeOH}$ for $3 \mathrm{~d}$, followed by vacuum activation. Energy-dispersed X-ray spectroscopy (EDX) confirmed an atomic ratio of 1:0.21 ( $\mathrm{Zr}: \mathrm{Fe})$ in these metal-loaded samples (Figure S7). Based on ${ }^{1} \mathrm{H}$ NMR integration showing $\sim 31 \%$ of the organic linkers are catbdc (overall formula $\mathrm{Zr}_{6} \mathrm{O}_{4}(\mathrm{OH})_{4}(\mathrm{bdc})_{4.1}(\text { catbdc })_{1.9}$, Figure 1) the measured metal ratio suggests $\sim 68 \%$ of the catbdc ligands are metalated. Importantly, no $\mathrm{Cl}$ was detected by $\mathrm{EDX}$, indicating that free or weakly bound $\mathrm{Fe}\left(\mathrm{ClO}_{4}\right)_{3}$ species were removed by washing. Again, PXRD and SEM confirmed retention of the UiO-66 crystallinity and phase purity (Figures S1 and S8). A BET surface area of $1134 \pm 67 \mathrm{~m}^{2} / \mathrm{g}$ was obtained using $\mathrm{N}_{2}$ gas at $77 \mathrm{~K}$, which supports that the $\mathrm{Fe}$ (III) ions are bound to the framework and not forming particles or otherwise blocking the pores of the MOF.

Fe K-edge extended X-ray absorption fine structure spectroscopy (EXAFS) was performed on UiO-66-FeCAT to investigate the coordination environment of the $\mathrm{Fe}$ (III) centers on the strut of UiO-66 framework. The data were best fit using an octahedral geometry at the $\mathrm{Fe}^{3+}$ center, with bidentate coordination from the catecholato ligand and the remainder of the coordination sphere being comprised of four solvent molecules that were best fit as three methanol (gave a better fit than water) and one methoxide ligand (based on required charge balance for $\mathrm{Fe}^{3+}$, Figure 4). This appears to be a
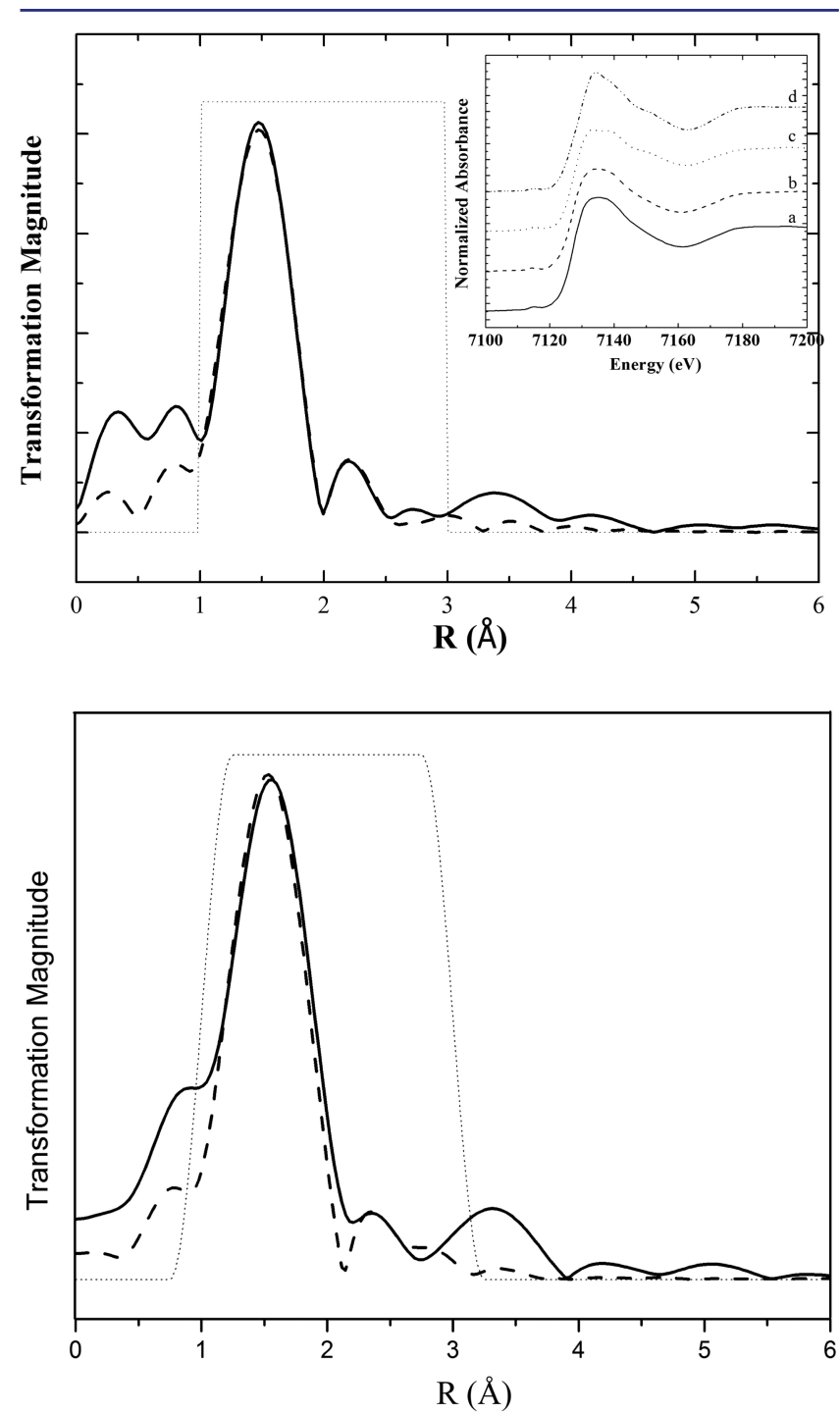

Figure 4. Fourier transformed EXAFS spectrum of UiO-66-FeCAT (top) and UiO-66-CrCAT (bottom). The black solid line is the experimental data, the dashed line is the best fit to data, and the dotted line is fitting window. Inset in top figure: XANES of UiO-66-FeCAT metalated by $\mathrm{Fe}\left(\mathrm{ClO}_{4}\right)_{3}$ (a, solid line), UiO-66-FeCAT metalated by $\mathrm{Fe}\left(\mathrm{CF}_{3} \mathrm{SO}_{3}\right)_{3}$ (b, dashed line), $\mathrm{Fe}\left(\mathrm{ClO}_{4}\right)_{3}$ (c, dotted line), and $\mathrm{Fe}\left(\mathrm{CF}_{3} \mathrm{SO}_{3}\right)_{3}$ (d, dashed dotted line).

reasonable formulation as a variety of $\mathrm{Fe}^{3+}$-based complexes with a methoxide ligand have been studied and crystallographically characterized. ${ }^{36}$ The data fitting indicated $\mathrm{Fe}^{3+}$ bonds to two oxygen atoms from the catecholato ligand at a distance of 1.98(3) $\AA$ and two nearest-neighbor carbon atoms from the catecholato ligand at a distance of 2.60(5) $\AA$. These distances are in good agreement with crystallographic data of $\mathrm{Fe}^{3+}$-catecholato species. ${ }^{37,38}$ In order to further characterize the binding of $\mathrm{Fe}^{3+}$ by catechol, we employed another metal precursor $\mathrm{Fe}\left(\mathrm{CF}_{3} \mathrm{SO}_{3}\right)_{3}$ to metalate UiO-66-CAT (via PSD) under identical conditions as used for $\mathrm{Fe}\left(\mathrm{ClO}_{4}\right)_{3}$. X-ray absorption near-edge structure (XANES) shows both $\mathrm{Fe}$ $\left(\mathrm{ClO}_{4}\right)_{3}$ and $\mathrm{Fe}\left(\mathrm{CF}_{3} \mathrm{SO}_{3}\right)_{3}$ treated $\mathrm{UiO}-66-\mathrm{CAT}$ (via PSD) display similar chemical environments around $\mathrm{Fe}^{3+}$, even 
though the iron precursors are readily distinguishable by XANES (Figure 4 top). Also, EDX of metal-loaded samples via $\mathrm{Fe}\left(\mathrm{CF}_{3} \mathrm{SO}_{3}\right)_{3}$ suggest negligible remaining sulfur but a similar atomic ratio of $\mathrm{Zr}: \mathrm{Fe}(1: 0.18)$ as $\mathrm{Fe}\left(\mathrm{ClO}_{4}\right)$-treated $\mathrm{UiO}-66$ CAT (via PSD). Finally, formation of an isolated monocatecholato $\mathrm{Fe}^{3+}$ species in UiO-66-FeCAT was confirmed by diffuse reflectance solid-state UV-vis spectroscopy (Figure S9). UiO-66-FeCAT exhibited a characteristic broad absorption band in the range of $\sim 700-750 \mathrm{~nm}$, characteristic of monocatecholato $\mathrm{Fe}^{3+}$ species. ${ }^{39,40}$ In contrast, both $\mathrm{Fe}^{3+}$ precursors and UiO-66-CAT (via PSD) did not show any significant absorption $>600 \mathrm{~nm}$.

The iron centers in UiO-66-FeCAT were further characterized by electron paramagnetic resonance (EPR) and ${ }^{57} \mathrm{Fe}$ Mössbauer spectroscopy. Variable-temperature X-band EPR measurements $(9-289 \mathrm{~K})$ on solid-samples of UiO-66-FeCAT (Figures 5, S10, and Figure S11) show a rhombic $S=5 / 2$ signal
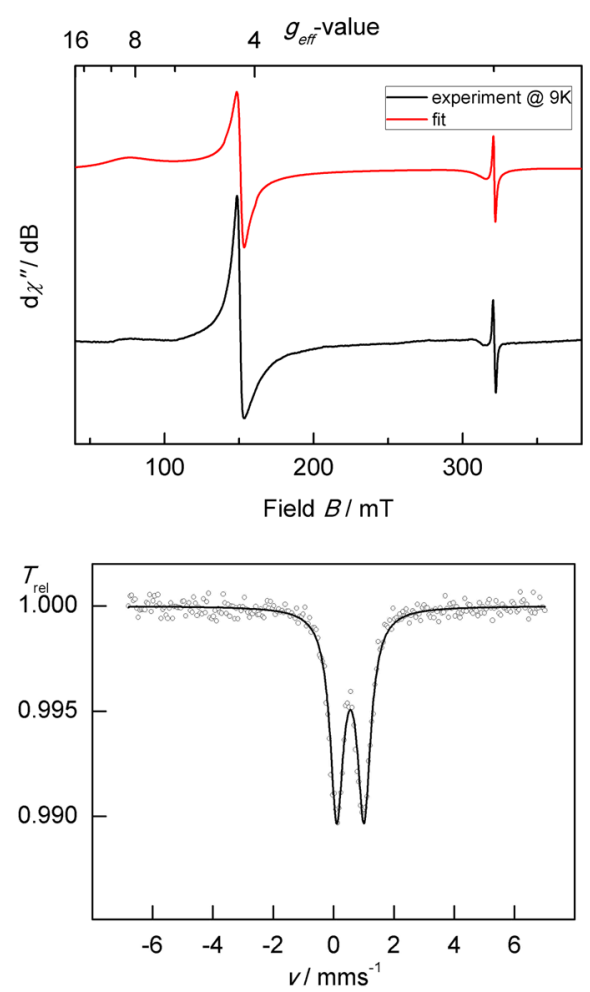

Figure 5. EPR spectrum of UiO-66-FeCAT (top) recorded in solid state at $9 \mathrm{~K}$ with simulation. Frequency $8.987052 \mathrm{GHz}$, power 0.3 $\mathrm{mW}$, modulation width $2 \mathrm{mT}$, time constant $0.1 \mathrm{~s}$. Parameters for simulation: Lorentzian line shape; species 1 (99.8\%): $g_{1}=2.02, g_{2}=$ $4.25, g_{3}=8.80$; species 2 (0.2\%): $g=1.997$. Mössbauer spectrum of UiO-66-FeCAT (bottom), recorded (for 8 days) at $77 \mathrm{~K}: \delta=0.54(1)$ $\mathrm{mms}^{-1}, \Delta E_{\mathrm{Q}}=0.91(1) \mathrm{mms}^{-1}, \Gamma_{\text {fwhm }}=0.54(1) \mathrm{mms}^{-1}$.

with $g$-values at 8.80, 4.25, and 2.02. This signal accounts for $99.8 \%$ of the total signal intensity. In addition, a sharp isotropic signal is observed at $g=1.997$ ( $0.2 \%$ intensity), which may originate from a minute semiquinone radical impurity $(S=1 / 2)$ from the catbdc ligand. The major $S=5 / 2$ signal confirms the expected $\mathrm{Fe}(\mathrm{III}) \mathrm{d}^{5}$ high-spin state of the metal centers incorporated in UiO-66-FeCAT. ${ }^{41}$ In addition to EPR, a zerofield ${ }^{57} \mathrm{Fe}$ Mössbauer spectrum of UiO-66-FeCAT recorded at $77 \mathrm{~K}$ in the solid-state of an identical sample (containing naturally abundant ${ }^{57} \mathrm{Fe}$ ) shows a single, well-resolved quadrupole doublet, further proving the existence of one distinct iron species in $\mathrm{UiO}-66-\mathrm{FeCAT}$ (Figure 5). Data fitting results in an isomer shift $(\delta)$ of $0.54(1) \mathrm{mm} \cdot \mathrm{s}^{-1}$ and a quadrupole splitting parameter, $\Delta E_{\mathrm{Q}}$, of $0.91(1) \mathrm{mm} \cdot \mathrm{s}^{-1}$. These values are characteristic of high-spin Fe(III) species and are in good agreement with published values for octahedral high-spin $\mathrm{Fe}$ (III) catecholate complexes. ${ }^{42-44}$ Accordingly, EPR and Mössbauer spectroscopy supports the proposed structural motif determined by EXAFS and diffuse reflectance solid-state UVvis spectroscopy.

Metalation of UiO-66-CAT with Cr. UiO-66-CAT prepared via the PSE approach described above (Scheme 1) was metalated in a postsynthetic manner with $\mathrm{Cr}$. Metalation of the catechol unit in UiO-66-CAT ( $51 \%$ catbdc) was carried out in aqueous $\mathrm{K}_{2} \mathrm{CrO}_{4}$ solution under weakly acidic condition ( $\mathrm{pH}$ $=3$ ). After $24 \mathrm{~h}$, the resulting dark-brown solids were isolated by centrifugation and washed by copious amount of deionized water until the supernatant was colorless. Followed by immersion in fresh $\mathrm{MeOH}$ for $3 \mathrm{~d}$ and vacuum drying, $\mathrm{UiO}$ 66-CrCAT was found to be highly crystalline and phase pure by PXRD and SEM (Figures S12 and S13). EDX suggests an 1:0.55 atomic ratio of $\mathrm{Zr}: \mathrm{Cr}$, indicating a nearly quantitative metalation of catechol units in the framework with overall formula as $\mathrm{Zr}_{6} \mathrm{O}_{4}(\mathrm{OH})_{4}(\mathrm{bdc})_{3}(\mathrm{Crcatbdc})_{3}$. The slightly higher $\mathrm{Cr}$ incorporation is likely due to trapped $\mathrm{K}_{2} \mathrm{CrO}_{4}$ in the UiO-66 lattice. However, a very low potassium (atomic ratio $\mathrm{Cr}: \mathrm{K}=$ 1:0.07) was observed in EDX, indicating a vast majority of $\mathrm{Cr}$ was strongly bound with the catechol sites. A control experiment was performed by incubation of unfunctionalized UiO-66 in aqueous $\mathrm{K}_{2} \mathrm{CrO}_{4}$ solution under the same condition (Figure S14). After extensive washing with fresh $\mathrm{H}_{2} \mathrm{O}$ and $\mathrm{MeOH}, \mathrm{EDX}$ of resulting solids confirms the presence of trapped potassium and chromium, with an atomic ratio of 1:0.04 ( $\mathrm{Zr}: \mathrm{Cr}$ ). The BET surface area of UiO-66-CrCAT was $708 \pm 24 \mathrm{~m}^{2} / \mathrm{g}\left(\mathrm{N}_{2}\right.$ at $\left.77 \mathrm{~K}\right)$, which is somewhat lower than the pristine UiO-66 $\left(1403 \pm 28 \mathrm{~m}^{2} / \mathrm{g}\right)$, likely due to the higher degree of functionality and metalation than the iron samples described above.

X-ray photoelectron spectroscopy (XPS) of UiO-66-CrCAT consists of two $\mathrm{Cr}$ peak contributions 587 and $577.2 \mathrm{eV}$ corresponding to $2 \mathrm{p}^{1 / 2}$ and $2 \mathrm{p}^{3 / 2}$ energy levels, respectively. These values compare well with binding energies in $\mathrm{Cr}\left(\mathrm{NO}_{3}\right)_{3}$ $\left(2 \mathrm{p}^{1 / 2} 587 \mathrm{eV}\right.$ and $\left.2 \mathrm{p}^{3 / 2} 577.3 \mathrm{eV}\right)$; however, they are distinctly different from $\mathrm{K}_{2} \mathrm{CrO}_{4}\left(2 \mathrm{p}^{1 / 2} 589 \mathrm{eV}\right.$ and $2 \mathrm{p}^{3 / 2} 579.9 \mathrm{eV}$, Figure $\mathrm{S} 15)$. This suggested that $\mathrm{Cr}(\mathrm{VI})$ is reduced to $\mathrm{Cr}(\mathrm{III})$ during the acidic metalation reaction conditions. Therefore, we tentatively assigned the metalated species as $\mathrm{Cr}^{3+}$ centers with an octahedral coordination environment, bound to the catecholato ligand with four weakly bound solvent molecules and/or counteranion. In order to confirm this coordination, $\mathrm{Cr}$ K-edge EXAFS was performed on UiO-66-CrCAT to study the coordination environment of the $\mathrm{Cr}^{3+}$ sites. A search in Cambridge structure database does not give a deposited crystal structure with a free methanol solvent molecule bound to a $\mathrm{Cr}$ catechol complex. Instead, we used a structure with a bidentate catecholato ligand along with three solvent water molecules and one hydroxide anion (required for charge balance) to perform the EXAFS data fitting. Fitting with this model gives a reasonable $R$-factor of 0.0170 (Figure 4 bottom). The data fitting gave atomic distance between $\mathrm{Cr}^{3+}$ and two oxygen atoms of 1.97(6) $\AA$ and an extended bond length between $\mathrm{Cr}^{3+}$ to solvent water molecules at 2.03(4) $\AA$. Both distances are in good agreement with crystallographic data of known $\mathrm{Cr}^{3+}$ catecholato structures. ${ }^{45}$ The slight disagreement between the 
experimental data and fit may be due to a small amount of chromate trapped inside the MOFs (e.g., advantageous binding not bound to the catecholato centers, see EDX data above) as well as the possible presence of $\mathrm{MeOH}$ as solvent molecules bound to some of the $\mathrm{Cr}^{3+}$ centers. The hypothesis that a trace amount of chromate may be contributing to the data is also evidenced by XANES, which shows a characteristic $\mathrm{Cr}^{6+}$ preedge feature from $1 s-3 \mathrm{~d}$ electronic transition (Figure S16). ${ }^{46}$

Oxidation Catalysis. Ketones are among the most important chemicals as final products or as intermediates in the pharmaceutical and chemical industry. ${ }^{47}$ The liquid-phase oxidation of secondary alcohols is the most widely used synthetic method to produce ketones. However, the classical approach for this reaction requires stoichiometric amount of $\mathrm{Cr}^{6+}$ or $\mathrm{Mn}^{7+}$ oxidants (e.g., Jones oxidation), thus generating equal amounts of metal waste that is environmentally disadvantageous. ${ }^{48}$ In a constant search for greener methods, many catalytic systems based on expensive noble metals, with high catalyst loadings, and costly/toxic additives have been explored. ${ }^{49,50}$ Both homogeneous and heterogeneous catalytic systems have been developed as well as metal nanoparticles; however, heterogeneous catalysts are preferable in industry due to their easy separation and reusability. ${ }^{51}$ High-valent $\mathrm{Cr}$ species are among the most extensively studied catalysts for oxidation reactions. ${ }^{52}$ Efforts were made to prepare heterogeneous catalysts with $\mathrm{Cr}$ active sites via $\mathrm{Cr}$-exchanged molecular sieves (i.e., aluminophosphates and silicates); ${ }^{53}$ however, leaching of $\mathrm{Cr}$ from the zeolite matrix during liquid-phase oxidation presented a problem. ${ }^{54}$

Utilizing MOFs as heterogeneous alcohol oxidation catalysts with hydroperoxides or molecular oxygen has gained increasing attention for green chemistry processes. ${ }^{55,56}$ Noble-metal nanoparticles encapsulated within MOFs also exhibit efficient aerobic oxidation of alcohol. ${ }^{57-59}$ HKUST-1 (with the formula $\mathrm{Cu}_{3}(\mathrm{BTC})_{2}$, where BTC $=1,3,5$-benzene tricarboxylate) in the presence of TEMPO under basic conditions gives moderate activity for the oxidation of benzylic alcohols but shows poor activity for oxidation of aliphalic, secondary, and cyclic alcohols. ${ }^{60}$ PSM of open bipyridine sites in MOF-253 with an organometallic $\mathrm{Ru}$ complex gives efficient alcohol oxidation with $\mathrm{PhI}(\mathrm{OAc})_{2}$ as the oxidant. ${ }^{61}$ Another $\mathrm{Cu}$-based MOF, constructed with paddlewheel secondary-building units (SBUs) and a 1,4-cyclohexanedicarboxylate linker, was found to have moderate activity ( 10 to $\sim 55 \%$ yield) for both primary and secondary alcohol oxidation with $\mathrm{H}_{2} \mathrm{O}_{2}{ }^{62}$ Herein, UiO-66CrCAT is shown to be a rare example of a heterogeneous catalysts containing nonprecious metal active centers that are efficient, clean, and recyclable for secondary alcohol oxidation with less polluting sacrificial oxidants (e.g., TBHP or $\mathrm{H}_{2} \mathrm{O}_{2}$ ) for "green" chemistry catalysis.

Secondary alcohol oxidation catalysis using UiO-66-CrCAT was initially studied with 2-heptanol as the substrate using 1.3 equiv of TBHP as an oxidant and chlorobenzene as the solvent at $70{ }^{\circ} \mathrm{C}$. Using UiO-66-CrCAT (only $1 \mathrm{~mol} \% \mathrm{Cr}$ ) gave nearly quantitative oxidation to 2-heptanone in $24 \mathrm{~h}$. More importantly, performing the catalysis in a "green," solvent-free environment significantly enhanced the rate of reaction, with the same oxidation complete in $8 \mathrm{~h}$ using only $0.5 \mathrm{~mol} \% \mathrm{Cr}$ loading. Meanwhile, the same mol \% of unfunctionalized UiO66 and UiO-66-CAT (via PSE without metalation) only gave $8 \%$ and $12 \%$ yield of 2 -heptanone after $24 \mathrm{~h}$, respectively. In addition, UiO-66 treated with $\mathrm{K}_{2} \mathrm{CrO}_{4}$ solution, rinsed, and used as a catalyst only gave $\sim 12 \%$ yield, indicating that trapped
$\mathrm{K}_{2} \mathrm{CrO}_{4}$ or other $\mathrm{Cr}$ species is not the source of the high catalytic activity of UiO-66-CrCAT. Moreover, $\mathrm{Cr}(\mathrm{acac})_{3}$ (acac $=$ acetylacetonate) and $\mathrm{K}_{2} \mathrm{CrO}_{4}$ were used as homogeneous catalysts; however, $1.5 \mathrm{~mol} \% \mathrm{Cr}$ of either compound gave $<40 \%$ yield after $24 \mathrm{~h}$. Another homogeneous control was carried out using a combination of $1.5 \mathrm{~mol} \%$ of $\mathrm{K}_{2} \mathrm{CrO}_{4}$ and $1.5 \mathrm{~mol} \%$ catbdc as catalyst, which also produced $<40 \%$ yield under the same reaction condition.

Table 1. Cr-Catalyzed Oxidation of 2-Heptanol ${ }^{a}$

\begin{tabular}{|c|c|c|c|c|c|c|}
\hline Entry & Catalyst & $\mathrm{Cr}(\mathrm{mol} \%)$ & $\operatorname{MOF}(\mathrm{mol} \%)$ & Solvent & Time (h) & Yield $(\%)^{[b]}$ \\
\hline 1 & blank & 0 & 0 & $\mathrm{C}_{6} \mathrm{H}_{5} \mathrm{Cl}$ & 24 & 8 \\
\hline 2 & UiO-66 & 0 & 2 & $\mathrm{C}_{6} \mathrm{H}_{5} \mathrm{Cl}$ & 24 & 8 \\
\hline 3 & UiO-66-CAT & 0 & 2 & $\mathrm{C}_{6} \mathrm{H}_{5} \mathrm{Cl}$ & 24 & 12 \\
\hline 4 & $\mathrm{UiO}-66, \mathrm{~K}_{2} \mathrm{CrO}_{4}{ }^{[\mathrm{c}]}$ & 0.08 & 2 & $\mathrm{C}_{6} \mathrm{H}_{5} \mathrm{Cl}$ & 24 & 12 \\
\hline 5 & $\mathrm{~K}_{2} \mathrm{CrO}_{4}$ & 1.5 & N/A & $\mathrm{C}_{6} \mathrm{H}_{5} \mathrm{Cl}$ & 24 & 35 \\
\hline 6 & $\mathrm{Cr}(\mathrm{acac})_{3}$ & 1.5 & N/A & $\mathrm{C}_{6} \mathrm{H}_{5} \mathrm{Cl}$ & 24 & 28 \\
\hline 7 & $\mathrm{~K}_{2} \mathrm{CrO}_{4} /$ catbdc & 1.5 & N/A & $\mathrm{C}_{6} \mathrm{H}_{5} \mathrm{Cl}$ & 24 & 35 \\
\hline 8 & UiO-66-CrCAT & 1 & 2 & $\mathrm{C}_{6} \mathrm{H}_{5} \mathrm{Cl}$ & 24 & 99 \\
\hline 9 & UiO-66-CrCAT & 0.5 & 1 & neat & 8 & 99 \\
\hline
\end{tabular}

${ }^{a}$ Reaction conditions: $1 \mathrm{mmol}$ 2-heptanol; $1.3 \mathrm{mmol}$ TBHP in $1 \mathrm{~mL}$ chlorobenzene (or neat) at $70{ }^{\circ} \mathrm{C} .{ }^{b}$ Based on GC-MS analysis. ${ }^{c} \mathrm{UiO}$ 66 was treated with $\mathrm{K}_{2} \mathrm{CrO}_{4}$ at $\mathrm{pH}=3$ and rinsed extensively with fresh $\mathrm{H}_{2} \mathrm{O}$ and $\mathrm{MeOH}$ before testing as a catalyst.

Eight secondary alcohols, including aliphatic, cyclic, benzylic, and a steroid alcohol were used as substrates for oxidation (Table 2). Among them, the majority of substrates gave nearly quantitative yields in 8 to $24 \mathrm{~h}$ using $1 \mathrm{~mol} \% \mathrm{Cr}$ in the form of UiO-66-CrCAT. Catalysis under solvent-free conditions gave comparable yields with the same catalyst loadings. Control reactions of catalytic amount of homogeneous $\mathrm{Cr}$ catalysts $\left(\mathrm{Cr}(\mathrm{acac})_{3}\right.$ or $\mathrm{K}_{2} \mathrm{CrO}_{4}$ ) and $\mathrm{UiO}-66-\mathrm{CAT}$ (via PSE without metalation) under the same conditions gave significantly lower yields $(<50 \%)$. Compared to other Cr-based heterogeneous catalysts, the MOF system described here is significantly improved. For example, a Cr-exchanged zeolite catalyst ${ }^{53}$ gave yields of 79-95\% with benzylic alcohols but required 4 equiv of TBHP. ${ }^{57}$ Moreover, UiO-66-CrCAT shows no leaching of $\mathrm{Cr}$, which was also a limitation for the zeolite catalyst. When compared to other MOF systems, the turnover frequencies (TOFs) of UiO-66-CrCAT are lower than systems that use precious-metal nanoparticles embedded in MOFs. Also, when compared to a system employing $\mathrm{Ru}$ and a more toxic oxidant ( $\left.\mathrm{PhI}(\mathrm{OAc})_{2}\right)$, UiO-66-CrCAT gives comparable yields but at $\sim 10$-fold less TOF. ${ }^{61}$ However, the yields and TOFs of $\mathrm{UiO}$ 66-CrCAT are significantly higher than other MOFs that use nonprecious metal catalysts, such as $\mathrm{Cu}$ or $\mathrm{Co}^{62}$ Although some of nonprecious metal homogeneous catalysts (mostly copper based) ${ }^{63}$ can give good yields using air or $\mathrm{O}_{2}$ as the oxidant, they often require much higher metal loadings (5$10 \%)^{64}$ and toxic/costly additives (e.g., TEMPO ${ }^{65}$ or dialkylazodicarboxylate $)^{66}$ as cocatalysts. In addition, efforts to produce heterogeneous versions of these copper catalysts are rather limited. 51,67

To test UiO-66-CrCAT with a complex substrate, $5 \alpha$ cholestan-3 $\beta$-ol was oxidized to the corresponding ketone in 12 $\mathrm{h}$ in nearly quantitative yield. The molecular size of this cholesterol derivative is approximately $16.4 \times 8.1 \times 6.5 \AA$ as determined by space-filling models (using the software package Diamond v3.1e). Although two dimensions of the substrate can fit within the pores of UiO-66 (pore diameters of $\sim 8 \AA$ for the 
Table 2. Cr-Catalyzed Oxidation of Secondary Alcohols Using TBHP (equiv listed) As Oxidants ${ }^{a}$

\begin{tabular}{|c|c|c|c|c|c|c|}
\hline Entry & Product & TBHP & Solvent & Time (h) & Yield $(\%)^{[b]}$ & TOF $\left(h^{-1}\right)^{[\mathrm{c}]}$ \\
\hline 1 & & 1.3 & $\mathrm{C}_{6} \mathrm{H}_{5} \mathrm{Cl}$ & 24 & 99 & $4.13(22.86)^{[\mathrm{d}]}$ \\
\hline 2 & & 1.3 & $\mathrm{C}_{6} \mathrm{H}_{5} \mathrm{Cl}$ & 24 & 92 & 3.84 \\
\hline 3 & & 1.3 & $\mathrm{C}_{6} \mathrm{H}_{5} \mathrm{Cl}$ & 24 & 70 & 2.94 \\
\hline 4 & & 1.3 & $\mathrm{C}_{6} \mathrm{H}_{5} \mathrm{Cl}$ & 24 & 95 & 4.02 \\
\hline 5 & & 1.1 & $\mathrm{C}_{6} \mathrm{H}_{5} \mathrm{Cl}$ & 12 & 99 & 8.34 \\
\hline 6 & & 1.1 & $\mathrm{C}_{6} \mathrm{H}_{5} \mathrm{Cl}$ & 12 & 99 & 8.34 \\
\hline 7 & & 1.1 & $\mathrm{C}_{6} \mathrm{H}_{5} \mathrm{Cl}$ & 8 & 99 & 12.48 \\
\hline 8 & & 1.1 & $\mathrm{C}_{6} \mathrm{H}_{5} \mathrm{Cl}$ & 12 & 99 & 8.34 \\
\hline 9 & & 1.3 & no solvent & 8 & 99 & $12.48(34.02)^{[\mathrm{d}]}$ \\
\hline 10 & & 1.3 & no solvent & 24 & 99 & 4.20 \\
\hline 11 & & 1.3 & no solvent & 24 & 72 & 3.00 \\
\hline 12 & & 1.3 & no solvent & 24 & 99 & 4.20 \\
\hline 13 & & 1.1 & no solvent & 24 & 99 & 4.20 \\
\hline
\end{tabular}

${ }^{a}$ Reaction conditions: $1 \mathrm{mmol} \sec$-alcohol, 1.1 (or 1.3) mmol TBHP in $1 \mathrm{~mL}$ chlorobenzene (or neat) at $70{ }^{\circ} \mathrm{C}$. ${ }^{b}$ Based on GC-MS analysis. ${ }^{c}$ TOF calculated as mol(product) $/[\operatorname{mol}$ (catalyst) $\times$ (reaction time) $]$.

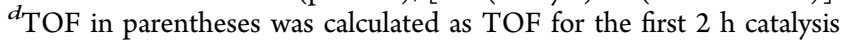
reaction.

tetrahedral cages and $\sim 11 \AA$ for the octahedral cages), the aperture size connecting these pore cages is only $\sim 6 \AA$, making interior access by $5 \alpha$-cholestan- $3 \beta$-ol unlikely. In light of this, absorption experiment was carried out between UiO-66-CAT (via PSE) and several substrates (2-heptanol, cyclooctanol, and 1-phenylethanol) at $70{ }^{\circ} \mathrm{C}$. After washing the surface of particles with methaol, digestion of the MOFs with dilute HF in $\mathrm{CD}_{3} \mathrm{OD}$ or $d^{6}$-DMSO revealed 2-heptanol and cyclooctanol inclusion in the MOFs (Figure S17). However, incubating the same MOF in saturated solution of $5 \alpha$-cholestan-3 $\beta$-ol in chlorobenzene under the same conditions did not show any substrate inclusion. We anticipate that $5 \alpha$-cholestan- $3 \beta$-ol is too large to diffuse into the pore cage and that catalysis with this substrate likely occurs on the surface of UiO particles. The minimal difference in yields and TOFs (Table 2) observed with different substrates suggests that catalysis maybe be occurring in both the interior and on the surface of the MOF with comparable efficiencies.

Compared with $t$-butyl hydroperoxide as an oxidant, catalysis with $\mathrm{H}_{2} \mathrm{O}_{2}$ is a more sustainable alternative, giving water as the sole byproduct. Alcohol oxidation was performed using 2 equiv of $30 \% \mathrm{H}_{2} \mathrm{O}_{2}$ in aqueous solution as the oxidant with acetonitrile as the solvent. Excellent yields (>95\%) were achieved for three examples of benzylic oxidation. However, attempts to expand to aliphatic alcohol oxidations were less encouraging, giving a yield of $\sim 39 \%$ for 2-heptanol. However, control reactions with $\mathrm{H}_{2} \mathrm{O}_{2}$ or TBHP alone (no MOF catalyst) gave yields of 0 and $8 \%$, respectively, showing that UiO-66CrCAT is still promoting this reaction.

Table 3. Cr-Catalyzed Oxidation of Secondary Alcohols Using $\mathrm{H}_{2} \mathrm{O}_{2}$ (equiv listed) As Oxidants ${ }^{a}$

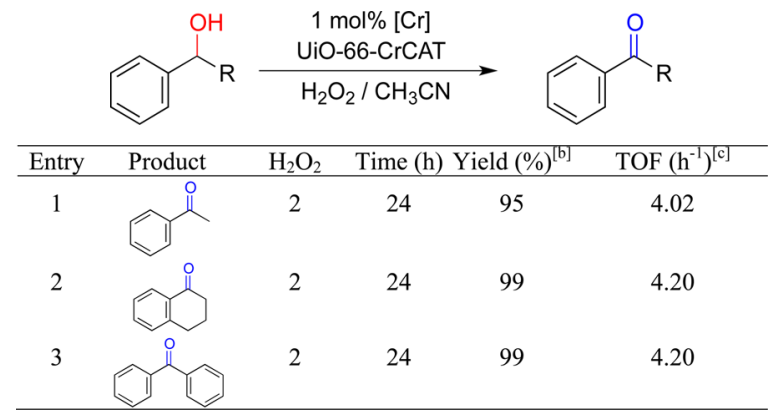

${ }^{a}$ Reaction conditions: $1 \mathrm{mmol}$ sec-alcohol; $2 \mathrm{mmol} \mathrm{H}_{2} \mathrm{O}_{2}$ in $1 \mathrm{~mL}$ $\mathrm{CH}_{3} \mathrm{CN}$ at $70{ }^{\circ} \mathrm{C}$. ${ }^{b}$ Based on GC-MS analysis. ${ }^{c} \mathrm{TOF}$ calculated as $\operatorname{mol}($ product $) /[\operatorname{mol}($ catalyst $) \times($ reaction time $)]$.

Heterogeneity of UiO-66-CrCAT was confirmed by a hot filtration of the catalysts after $2 \mathrm{~h}$ of reaction with 2-heptanol, which resulted in negligible additional yield of product up to 22 h after filtration (Figure S18). This suggested that UiO-66CrCAT was a true heterogeneous catalyst, and no catalytically active species were released into solution. The chemical robustness of UiO-66-CrCAT was confirmed by its incubation in aqueous solution for $24 \mathrm{~h}$ and its thermal stability up to $\sim 250{ }^{\circ} \mathrm{C}$ was also evidenced by thermogravimetric analysis (Figures S19 and S20). Leaching of Cr into the solution was examined by inductive coupled plasma-optical emission spectroscopy (ICP-OES, Quantitative Technologies Inc., Whitehouse, NJ), which showed that $<0.1 \mathrm{ppm} \mathrm{Cr}$ was present in the reaction solution. Overall, the robust UiO-66 material exhibited clean catalysis when compared to Cr-containing zeolites, which are often susceptible to solvolysis by polar solvents and extensive leaching of the metal species. ${ }^{54,68,69}$

To examine recyclability, experiments were performed using the same batch of $1 \mathrm{~mol} \% \mathrm{UiO}-66-\mathrm{CrCAT}$ for the oxidation of 2-heptanol over five runs. Between each run, the catalysts were simply separated by centrifugation, washed with $\mathrm{MeOH}$, and dried under vacuum at room temperature. No decrease of yield ( $\sim 99 \%)$ was observed between the five runs, highlighting the robust nature of the $\mathrm{Cr}$-grafted MOF catalyst. Characterization of UiO-66-CrCAT after catalysis showed the MOF still possessed high crystallinity as evidenced by PXRD and SEM (Figure S21). The disappearance of $\mathrm{Cr}^{6+}$ pre-edge features in the XANES spectrum after one round of catalysis indicated that the residual $\mathrm{Cr}^{6+}$ observed in freshly prepared UiO-66-CrCAT was removed or reduced to $\mathrm{Cr}^{3+}$ (Figure S20). Based on XPS and EXAFS analysis, no notable change in the coordination environment of the $\mathrm{Cr}$ centers (other than the aforementioned disappearance of trace $\mathrm{Cr}^{6+}$ ) was observed after one-cycle of 2heptanol oxidation (Figures S22 and S23), again suggesting that the catalytic species is highly robust.

In summary, the catalysis presented here are the first examples in MOFs exhibiting efficient, recyclable, and "green" alcohol oxidation with nonprecious metal active species. Excellent yields were achieved with eight different secondary alcohols, including aliphatic, cyclic, and benzylic alcohols. The oxidations were performed using a very low metal loading 
(0.5-1 mol \%), in some cases under neat conditions and also in some cases using $\mathrm{H}_{2} \mathrm{O}_{2}$ as an environmentally benign oxidant. Moreover, the robustness of the UiO-66 framework allowed for easy recovery and reuse without leaching of $\mathrm{Cr}$.

\section{CONCLUSION}

Our study employs two fundamentally different strategies (PSD, PSE) to synthesize the first highly robust MOF bearing isolated monocatecholato metal sites on the strut of the organic linkers. The results strongly suggest that postsynthetic approaches are facile and important functionalization methods to access MOFs that cannot be directly synthesized. The PSE strategy presented here is a rare postsynthetic example to introduce open metal sites, in a one-step reaction. Metalation of these catecholato groups resulted in unprecedented metalmonocatecholato species, and both Fe-monocatecholato and $\mathrm{Cr}$-monocatecholato moieties were evaluated by EXAFS. UiO66-CrCAT proved to be an efficient and "green" alcohol oxidation catalyst for a range of substrates. Complete heterogeneity and recyclability of our MOF catalysts overcome the problem of leaching in Cr-exchanged molecular sieves.

\section{ASSOCIATED CONTENT}

\section{S Supporting Information}

Experimental details of synthesis and catalysis and additional characterizations. This material is available free of charge via the Internet at http://pubs.acs.org.

\section{AUTHOR INFORMATION}

\section{Corresponding Author}

scohen@ucsd.edu

\section{Notes}

The authors declare no competing financial interest.

\section{ACKNOWLEDGMENTS}

This work was supported by a grant from the National Science Foundation, Division of Materials Research (DMR-1262226). The synchrotron X-ray absorption spectroscopy data were collected at Advanced Photon Source in Argonne National Laboratory on beamline 9-BM through the general user proposal program. We thank Dr. Y. Su (UC San Diego) for assistance with the mass spectrometry data and helpful discussion.

\section{REFERENCES}

(1) O’Keeffe, M.; Yaghi, O. M. Chem. Rev. 2012, 112, 675

(2) Sumida, K.; Rogow, D. L.; Mason, J. A.; McDonald, T. M.; Bloch,

E. D.; Herm, Z. R.; Bae, T.-H.; Long, J. R. Chem. Rev. 2012, 112, 724.

(3) Li, J.-R.; Sculley, J.; Zhou, H.-C. Chem. Rev. 2012, 112, 869.

(4) Yoon, M.; Srirambalaji, R.; Kim, K. Chem. Rev. 2012, 112, 1196.

(5) Kreno, L. E.; Leong, K.; Farha, O. K.; Allendorf, M.; Van Duyne, R. P.; Hupp, J. T. Chem. Rev. 2012, 112, 1105.

(6) Rocca, J. D.; Liu, D.; Lin, W. Acc. Chem. Res. 2011, 44, 957.

(7) Morris, R. E.; Wheatley, P. S. Angew. Chem., Int. Ed. 2008, 47, 4966.

(8) Bloch, E. D.; Queen, W. L.; Krishna, R.; Zadrozny, J. M.; Brown, C. M.; Long, J. R. Science 2012, 335, 1606.

(9) Horike, S.; Dinca, M.; Tamaki, K.; Long, J. R. J. Am. Chem. Soc. 2008, 130, 5854.

(10) Biswas, S.; Zhang, J.; Li, Z.; Liu, Y. Y.; Grzywa, M.; Sun, L.; Volkmer, D.; Van Der Vorrt, P. Dalton Trans. 2013, 42, 4730.

(11) Yang, Q.; Vaesen, S.; Ragon, F.; Wiersum, A. D.; Wu, D.; Lago, A.; Devic, T.; Martineau, C.; Taulelle, F.; Llewellyn, P. L.; Jobic, H.;
Zhong, C.; Serre, C.; Weireld, G. D.; Maurin, G. Angew. Chem., Int. Ed. 2013, 52, 10316.

(12) Hwang, Y. K.; Hong, D.-Y.; Chang, J.-S.; Jhung, S. H.; Seo, Y.K.; Kim, J.; Vimont, A.; Daturi, M.; Serre, C.; Ferey, G. Angew. Chem., Int. Ed. 2008, 47, 4144.

(13) Tanabe, K. K.; Cohen, S. M. Angew. Chem., Int. Ed. 2009, 48, 7424.

(14) Cohen, S. M. Chem. Rev. 2012, 112, 970.

(15) Demessence, A.; D’Alessandro, D. M.; Foo, M. L.; Long, J. R. J. Am. Chem. Soc. 2009, 131, 8784.

(16) Tanabe, K. K.; Allen, C. A.; Cohen, S. M. Angew. Chem., Int. Ed. 2010, 49, 9730.

(17) Shultz, A. M.; Farha, O. K.; Adhikari, D.; Sarjeant, A. A.; Hupp, J. T.; Nguyen, S. T. Inorg. Chem. 2011, 50, 3174.

(18) Karagiaridi, O.; Bury, W.; Sarjeant, A. A.; Stern, C. L.; Farha, O. K.; Hupp, J. T. Chem. Sci. 2012, 3, 3256.

(19) Burnett, B. J.; Barron, P. M.; Hu, C. H.; Choe, W. J. Am. Chem. Soc. 2011, 133, 9984.

(20) Karagiaridi, O.; Lalonde, M. B.; Bury, W.; Sarjeant, A. A.; Farha, O. K.; Hupp, J. T. J. Am. Chem. Soc. 2012, 134, 18790.

(21) Kim, M.; Cahill, J. F.; Su, Y.; Prather, K. A.; Cohen, S. M. Chem. Sci. 2012, 3, 126.

(22) Kim, M.; Cahill, J. F.; Fei, H.; Prather, K. A.; Cohen, S. M. J. Am. Chem. Soc. 2012, 18082.

(23) Fei, H.; Cahill, J. F.; Prather, K. A.; Cohen, S. M. Inorg. Chem. 2013, 52, 4011 .

(24) Li, T.; Kozlowski, M. T.; Doud, E. A.; Blakely, M. N.; Rosi, N. L. J. Am. Chem. Soc. 2013, 135, 11688.

(25) Jeong, S.; Kim, D.; Song, X.; Choi, M.; Park, N.; Lah, M. S. Chem. Mater. 2013, 25, 1047.

(26) Pierpont, C. G.; Lange, C. W. Prog. Inorg. Chem. 2007, 41.

(27) Dhungana, S.; Heggemann, S.; Heinisch, L.; Mollmann, U.; Boukhalfa, H.; Crumbliss, A. L. Inorg. Chem. 2001, 40, 7079.

(28) Powell, H. K. J.; Taylor, M. C. Aust. J. Chem. 1982, 35, 739.

(29) Weston, M. H.; Farha, O. K.; Hauser, B. G.; Hupp, J. T.; Nguyen, S. T. Chem. Mater. 2012, 24, 1292.

(30) Tanabe, K. K.; Siladke, N. A.; Broderick, E. M.; Kobayashi, T.; Goldston, J. F.; Weston, M. H.; Farha, O. K.; Hupp, J. T.; Pruski, M.; Mader, E. A.; Johnson, M. J. A.; Nguyen, S. T. Chem. Sci. 2013, 4, 2483.

(31) Weston, M. H.; Peterson, G. W.; Brown, M. A.; Jones, P.; Farha, O. K.; Hupp, J. T.; Nguyen, S. T. Chem. Commun. 2013, 49, 2995.

(32) Nguyen, H. G. T.; Weston, M. H.; Farha, O. K.; Hupp, J. T.; Nguyen, S. T. CrystEngComm 2012, 14, 4115.

(33) Nguyen, H. G. T.; Weston, M. H.; Sarjeant, A. A.; Gardner, D. M.; An, Z.; Carmieli, R.; Wasielewski, M. R.; Farha, O. K.; Hupp, J. T.; Nguyen, S. T. Cryst. Growth. Des. 2013, 2013, 3528.

(34) Allen, C. A.; Cohen, S. M. J. Mater. Chem. 2012, 22, 10188.

(35) Pullen, S.; Fei, H.; Orthaber, A.; Cohen, S. M.; Ott, S. J. Am. Chem. Soc. 2013, 135, 16997.

(36) Jonas, R. T.; Stack, T. D. P. J. Am. Chem. Soc. 1997, 119, 8566.

(37) Merkel, M.; Schnieders, D.; Baldeau, S. M.; Krebs, B. Eur. J. Inorg. Chem. 2003, 783.

(38) A, L.-T.; Duan, L.-M.; Xu, X.-T.; Liu, S.-Q. Chem. Res. Chin. Univ. 2009, 25, 273.

(39) Sever, M. J.; Wilker, J. J. Dalton Trans. 2004, 1061

(40) Abu-Nawwas, A. H.; Cano, J.; Christian, P.; Mallah, T.; Rajaraman, G.; Teat, S. J.; Winpenny, R. E. P.; Yukawa, Y. Chem. Commun. 2004, 314.

(41) Weisser, J. T.; Nilges, M. J.; Sever, M. J.; Wilker, J. J. Inorg. Chem. 2006, 45, 7736.

(42) Hider, R. C.; Howlin, B.; Miller, J. R.; Mohd-Nor, A. R.; Silver, J. Inorg. Chim. Acta 1983, 80, 51.

(43) Hider, R. C.; Mohd-Nor, A. R.; Silver, J.; Morrison, I. E. G.; Rees, L. V. C. J. Chem. Soc., Dalton Trans. 1981, 609.

(44) von Nussbaum, F.; Spiteller, P.; Ruth, M.; Steglich, W.; Wanner, G.; Gamblin, B.; Stievano, L.; Wagner, F. E. Angew. Chem., Int. Ed. 1998, 37, 3292.

(45) Shiren, K.; Tanaka, K. Inorg. Chem. 2002, 41, 5912. 
(46) Peterson, M. L.; Brown, G. E.; Parks, G. A.; Stein, C. L. Geochim. Cosmochim. Acta 1997, 61, 3399.

(47) Smiley, R. A.; jackson, H. L. Chemistry and the Chemical Industry; CRC Press: Boca Raton, FL, 2002.

(48) Tojo, G.; Fernandez, M. Oxidation of Alcohols to Aldehydes and Ketones; Springer: New York, 2010.

(49) Corma, A.; Garcia, H. Chem. Soc. Rev. 2008, 37, 2096.

(50) Mallat, T.; Baiker, A. Chem. Rev. 2004, 104, 3037.

(51) Parmeggiani, C.; Cardona, F. Green Chem. 2012, 14, 547.

(52) Muzart, J. Chem. Rev. 1992, 92, 113.

(53) Lounis, Z.; Riahi, A.; Djafri, F.; Muzart, J. Appl. Catal., A 2006, 309, 270.

(54) Lempers, H. E. B.; Sheldon, R. A. J. Catal. 1998, 175, 62.

(55) Maksimchuk, N. V.; Kovalenko, K. A.; Fedin, V. P.; Kholdeeva,

O. A. Chem. Commun. 2012, 48, 6812 .

(56) Dhakshinamoorthy, A.; Alvaro, M.; Garcia, H. Catal. Sci. Technol. 2011, 1, 856.

(57) Liu, H.; Liu, Y.; Li, Y.; Tang, Z.; Jiang, H. J. Phys. Chem. C 2010, $114,13362$.

(58) Ishida, T.; Nagaoka, M.; Akita, T.; Haruta, M. Chem.-Eur. J. 2008, 14, 8456.

(59) Chen, G.; Wu, S.; Liu, H.; Jiang, H.; Li, Y. Green Chem. 2013, $15,230$.

(60) Dhakshinamoorthy, A.; Alvaro, M.; Garcia, H. ACS Catal. 2011, $1,48$.

(61) Carson, F.; Agrawal, S.; Gustafsson, M.; Bartoszewicz, A.; Moraga, F.; Zou, X.; Martin-Matute, B. Chem.-Eur. J. 2012, 18, 15337.

(62) Kato, C. N.; Hasegawa, M.; Sato, T.; Yoshizawa, A.; Inoue, T.; Mori, W. J. Catal. 2005, 230, 226.

(63) Hoover, J. M.; Stahl, S. S. J. Am. Chem. Soc. 2011, 133, 16901.

(64) Davi, M.; Lebel, H. Org. Lett. 2009, 11, 41.

(65) Figiel, P. J.; Sibaouih, A.; Ahmad, J. U.; Nieger, M.; Raisanen, M. T.; Leskela, M.; Repo, T. Adv. Synth. Catal. 2009, 351, 2625.

(66) Marko, I. E.; Gautier, A.; Dumeunier, R.; Doda, K.; Philippart, F.; Brown, S. M.; Urch, C. J. Angew. Chem., Int. Ed. 2004, 43, 1588.

(67) Herbert, M.; Montilla, F.; Galindo, A. Dalton Trans. 2010, 39, 900.

(68) Sheldon, R. A.; Wallau, M.; Arends, I. W. C. E.; Schuchardt, U. Acc. Chem. Res. 1998, 31, 485.

(69) Arends, I. W. C. E.; Sheldon, R. A. Appl. Catal., A 2001, 175. 